\title{
OPEN CHANNEL FLOW VELOCITY PROFILES FOR DIFFERENT REYNOLDS NUMBERS AND ROUGHNESS CONDITIONS
}

\author{
Md Abdullah Al Faruque 1 , Scott Wolcott ${ }^{2}$, Joshua Goldowitz ${ }^{3}$, Teresa Wolcott ${ }^{4}$ \\ ${ }^{1}$ Assistant Professor, Civil Engineering Technology, Rochester Institute of Technology, New York, USA, \\ ${ }^{2}$ Professor \& Program Coordinator, Civil Engineering Technology, Rochester Institute of Technology, New York, \\ USA \\ ${ }^{3}$ Professor \& Undergraduate Coordinator, Environmental Sustainability Health \& Safety, Rochester Institute of \\ Technology, New York, USA \\ ${ }^{4}$ Lecturer, Civil Engineering Technology, Rochester Institute of Technology, New York, USA
}

\begin{abstract}
A series of laboratory tests were carried out to understand the extent of effect of roughness and Reynolds number on mean velocity in both outer and inner scaling. To this end, four different types of bed surface conditions (impermeable smooth bed, impermeable rough bed, permeable sand bed and impermeable distributed roughness) and two different Reynolds number $\left(\right.$ Re $_{h}=$ 47,500 and 31,000) were adopted in the study. Sand particles of median diameter of $2.46 \mathrm{~mm}$ were used to create the roughness. The results show that the mean velocities collapsed well for different Reynolds number and for all different bed surfaces. The maximum velocity for all flow conditions were observed below some distances from the free surface. The location of maximum velocity is seen to be dependent on both of roughness and Reynolds number. The smooth bed test data agrees well with the standard log law and collapses well in viscous sub layer and overlap region. The extent of collapses is found to be dependent on Reynolds number. Friction coefficient is noted to be dependent on both the Reynolds number and roughness.
\end{abstract}

Key Words: Open channel flow, Reynolds number, Roughness, mean velocity, friction coefficient, log law

\section{INTRODUCTION}

Frequent drastic changes of bed condition are often encountered in hydraulic engineering. The velocity distribution would be changed in both streamwise and wallnormal direction due to this sudden change of bed surface. The understanding of flow characteristics in different bed conditions is very important in hydraulic engineering practice. One example is the deposition of insoluble or sorbed contaminants into river bed due to uncontrolled disposal of municipal and industrial waste. Entrained contaminants deposited in the river bed can be resuspended and transported downstream by maritime river traffic, can then deposit in a new location affecting the ecology and fisheries of previously uncontaminated areas.

\section{LITERATURE REVIEW}

Kirkgöz and Ardiçhoğlu (1997) studied the flow progression from developing to fully developed flow. They noted that at the axis of a fully developed turbulent flow section the boundary layer extends to the water surface if the aspect ratio $b / h \geq 3$. Here $b$ is the width of channel and $h$ is the depth of flow. They didn't observe any velocity dip for channel centerline even for channel with aspect ratio as low as $b / h=3$. Balachandar and Patel (2002) indicated that the streamwise mean velocity profiles follow the well-known logarithmic law for a smooth surface, and the appropriate downward shift, for a rough surface. Tachie et al. (2003) used laser-Doppler anemometer (LDA) to measure velocity on a smooth and two geometrically different types of rough surfaces in an open channel and showed that roughness effects on the velocity field were similar to those observed in a zero-pressure gradient turbulent boundary layer, even though the boundary layer in an open channel flow is influenced by the free surface. They observed that surface roughness substantially increased the value of the wake parameter in comparison to the smooth wall value. Nezu (2005) correlated aspect ratio (width/depth ratio of flow, $\mathrm{b} / \mathrm{h}$ ) with the formation of secondary current and noted that the maximum velocity on the centerline occurred below the free surface for $\mathrm{b} / \mathrm{h}<5$ (velocity-dip phenomenon) indicated the presence of secondary currents generated due to the effect of side wall. He examined the critical value of $b / h$ and proposed that rectangular channels could be classified according to whether $\mathrm{b} / \mathrm{h}<(\mathrm{b} / \mathrm{h})_{\text {crit }}$ (narrow open channel) or $\mathrm{b} / \mathrm{h}>(\mathrm{b} / \mathrm{h})_{\text {crit }}$ (wide open channel), with $(\mathrm{b} / \mathrm{h})_{\text {crit }}=5$ for smooth channel.

Afzal et al. (2009) studied the effect of Reynolds number on the velocity characteristics of smooth open-channel flows. Their mean velocity profile in inner scaling showed that the extent of overlap with the log region increased with increasing Reynolds number.

The existing literature on Reynolds number effect on mean velocities was briefly reviewed. No definitive statement can be made as to the persistence of the effect of Reynolds number on the velocity profile. Only few researchers have used laser Doppler anemometer technology to study flow 
velocities. The present study investigates the mean velocities in an open channel flow using LDA for various values of Reynolds number.

\section{EXPERIMENTAL SETUP}

Experiments were carried out in a 9-m long rectangular open channel flume (cross-section $1100 \mathrm{~mm}$ wide by $920 \mathrm{~mm}$ deep) (Figure 1). The header tank upstream of the rectangular cross-section was $1.2 \mathrm{~m}$ square and $3.0 \mathrm{~m}$ deep. The nominal flow depth in the measurement region was 100 $\mathrm{mm}$, resulting in a width-to-depth ratio (b/h) of approximately 11 . This value of the aspect ratio is considered to be large enough to minimize the effect of secondary currents and the flow can be considered to be nominally two-dimensional (Nezu, 2005). The water was recirculated by two 15 -horsepower centrifugal pumps. The sidewalls and bottom of the flume were made of transparent tempered glass to facilitate velocity measurements using a laser Doppler anemometer (LDA). The flume is a permanent facility and the quality of flow has been confirmed in several previous studies (Faruque et al., 2006; Sarathi et al., 2008). The bottom slope of the flume was adjustable and for this study, it was kept horizontal. Two constant discharges of $720 \mathrm{GPM}$ and $450 \mathrm{GPM}$ were used.

Four different types of bed surface conditions were used in this study. The base case was a hydraulically smooth surface generated using a $3.7 \mathrm{~m}$ long polished aluminum plate spanning the entire width of flume (Figure 2a). Three different types of rough surfaces were used. Sand particles $\left(\mathrm{d}_{50}=2.46 \mathrm{~mm}, \sigma_{\mathrm{g}}=\sqrt{d_{84} / d_{16}}=1.24\right)$ were used to create the rough surfaces. To generate the first rough surface (designated as 'distributed roughness'), 18-mm wide sand strips were glued to the smooth aluminium plate alternating with $18-\mathrm{mm}$ wide smooth strips as shown in Figure $2 \mathrm{~b}$. The second roughness condition consisted of the same sand grains glued over the entire smooth surface as shown in Figure 2c (continuous roughness). In both cases the sand was affixed to the aluminum plate in a single grain layer. Third rough surface was generated using 200-mm thick and $3.7 \mathrm{~m}$ long uniform sand bed as shown in Figure 3. The flow conditions were maintained in such a manner that there was no initiation of sand movement. As a precaution, a sand trap was provided at the downstream of the bed to prevent any accidental transport of sand particles into the pump/piping assembly.

Two different Reynolds numbers were used for each bed condition. Reynolds numbers were chosen in order to maintain subcritical flow conditions (i.e., Froude numbers less than unity). Flow conditions correspond to values of Reynolds number, $\operatorname{Re}_{\mathrm{h}}=\mathrm{U}_{\mathrm{avg}} \mathrm{d} / \mathrm{v} \approx 47,500 \& 31,000$ and the corresponding Froude numbers are $\mathrm{F}_{\mathrm{r}}=\mathrm{U}_{\mathrm{avg}} /(\mathrm{gd})^{0.5} \approx 0.40 \&$ 0.24 respectively. Here, $U_{\text {avg }}$ is the average velocity, $d$ is the depth of flow, $g$ is the acceleration due to gravity and $v$ is the kinematic viscosity of the fluid. In the test sections, the measured variation of water surface elevation was less than $1 \mathrm{~mm}$ over a streamwise distance of $600 \mathrm{~mm}$ implying a negligible pressure gradient. Flow straightners were used at the beginning and the end of flume to condition the flow. To ensure the presence of a turbulent boundary layer, a 3-mm diameter rod was used as a (Figure 1) trip. The boundary layer shape factor for the smooth bed defined as the ratio of displacement to momentum thickness was found to be $\approx 1.3$, which is an indication of fully developed turbulent flow (Schlichting, 1979). The measurements for the distributed roughness were conducted on top of the $60^{\text {th }}$ sand strip. All the measurements were conducted along the centreline of the channel to minimize secondary flow effects. Preliminary tests were conducted to ensure a fully developed flow condition.

A commercial two-component fibre-optic LDA system (Dantec Inc.) powered by a 300-mW Argon-Ion laser was used for the velocity measurements. This system has been used in several previous studies and details are avoided for brevity (Faruque et al., 2006; Bey et al., 2007; Afzal et al., 2009). The optical elements include a Bragg cell, a 500-mm focusing lens and the beam spacing was $38 \mathrm{~mm}$. 10,000 validated samples were acquired at each measurement location.

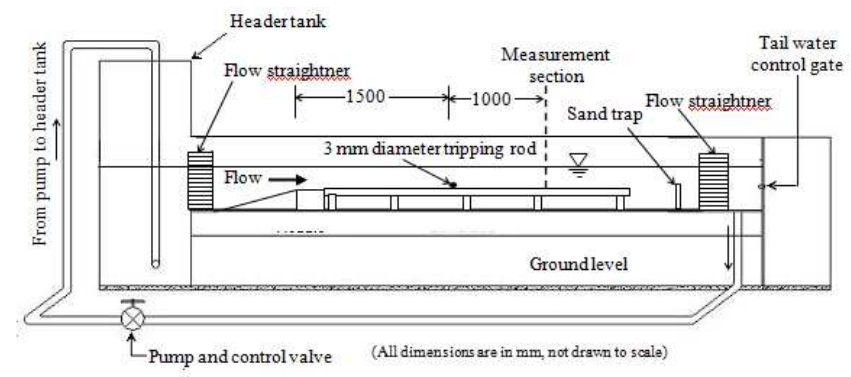

Figure 1: Schematic of the Open Channel Flume

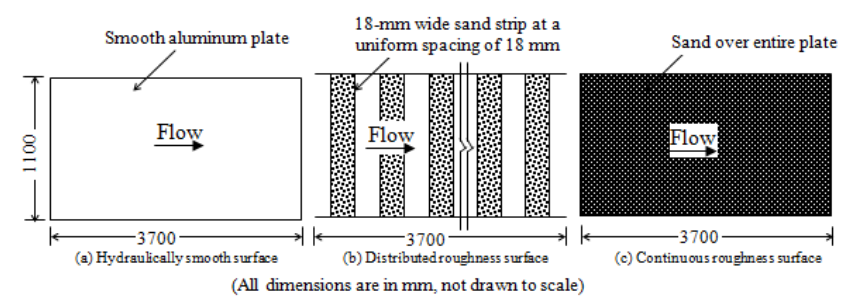

Figure 2: Plan view of different fixed bed condition.

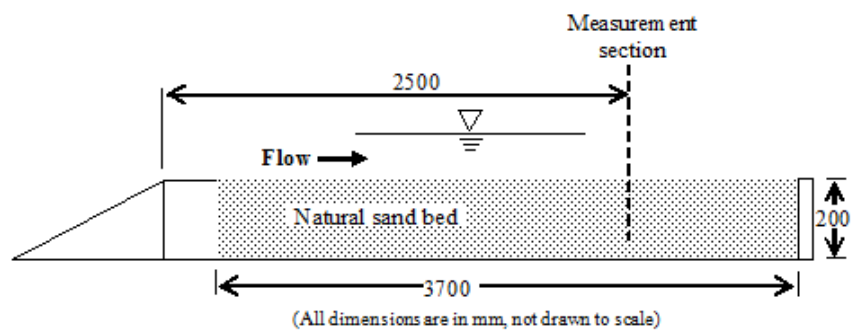

Figure 3: Section of natural sand bed.

\section{RESULTS AND DISCUSSION}

\subsection{Mean Velocity Profile: Outer Scaling}

The distributions of the streamwise component of the mean velocity in outer scaling are shown in Figure 4. Maximum velocity $\left(\mathrm{U}_{\mathrm{e}}\right)$ and maximum flow depth $(\mathrm{d})$ are used to nondimensionalize the mean velocity (U) and the wall normal 
distance (y), respectively. Figure 4a indicates that mean streamwise velocity for flow over a smooth bed collapses reasonably for different Reynolds number. Schlichting (1979) indicates that velocity profile becomes fuller as the Reynolds number increases in flow through smooth pipes. One can also note from the insets of Figure 4a that higher Reynolds number causes the increased velocity in the near bed location and simultaneously causes the reduction in velocity near the free surface to satisfy the continuity considerations. Similar observations of increased velocity in the near bed location and simultaneously causes the reduction in velocity near the free surface can be seen for the flow over the sand bed (Insets of Figure 4b). However, there is no effect of Reynolds number for flow near the bed or near the free surface for the flow over the distributed roughness (Insets of Figure 4c) and continuous roughness (Insets of Figure 4d). In the outer region, each velocity profile shows a slight dip where the local maximum value $\left(\mathrm{U}_{\mathrm{e}}\right)$ occurs below the free surface and $\delta \mathrm{U} / \delta \mathrm{y}$ is negative in the vicinity of the free surface. Although the velocity dip is largest for the sand bed (Figure 4b), the smooth surface shows a greater dip (Figure 4a) than both the continuous roughness (Figure 4d) and the distributed roughness (Figure $4 c)$.

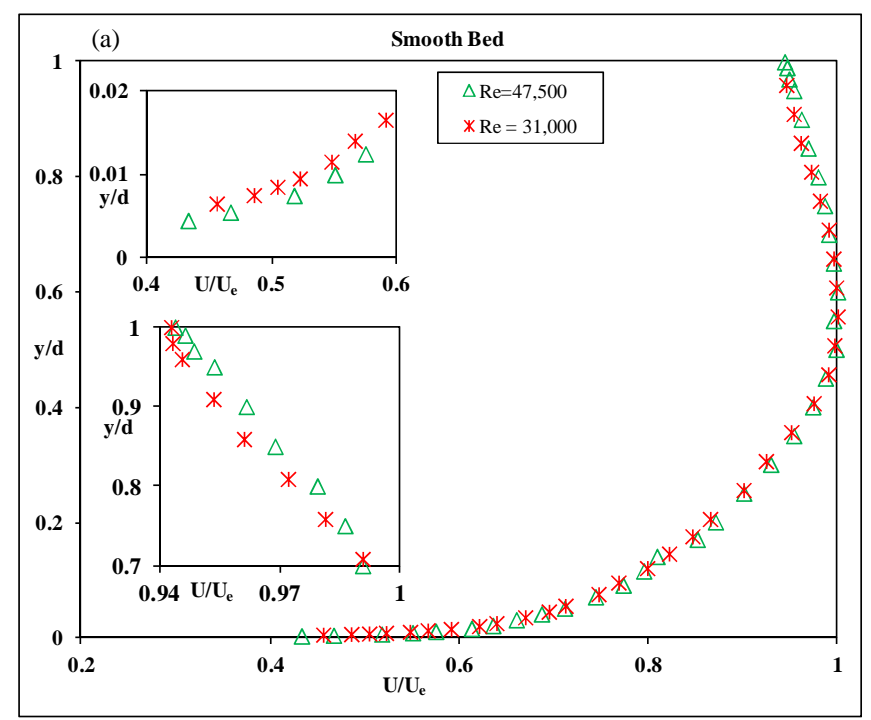

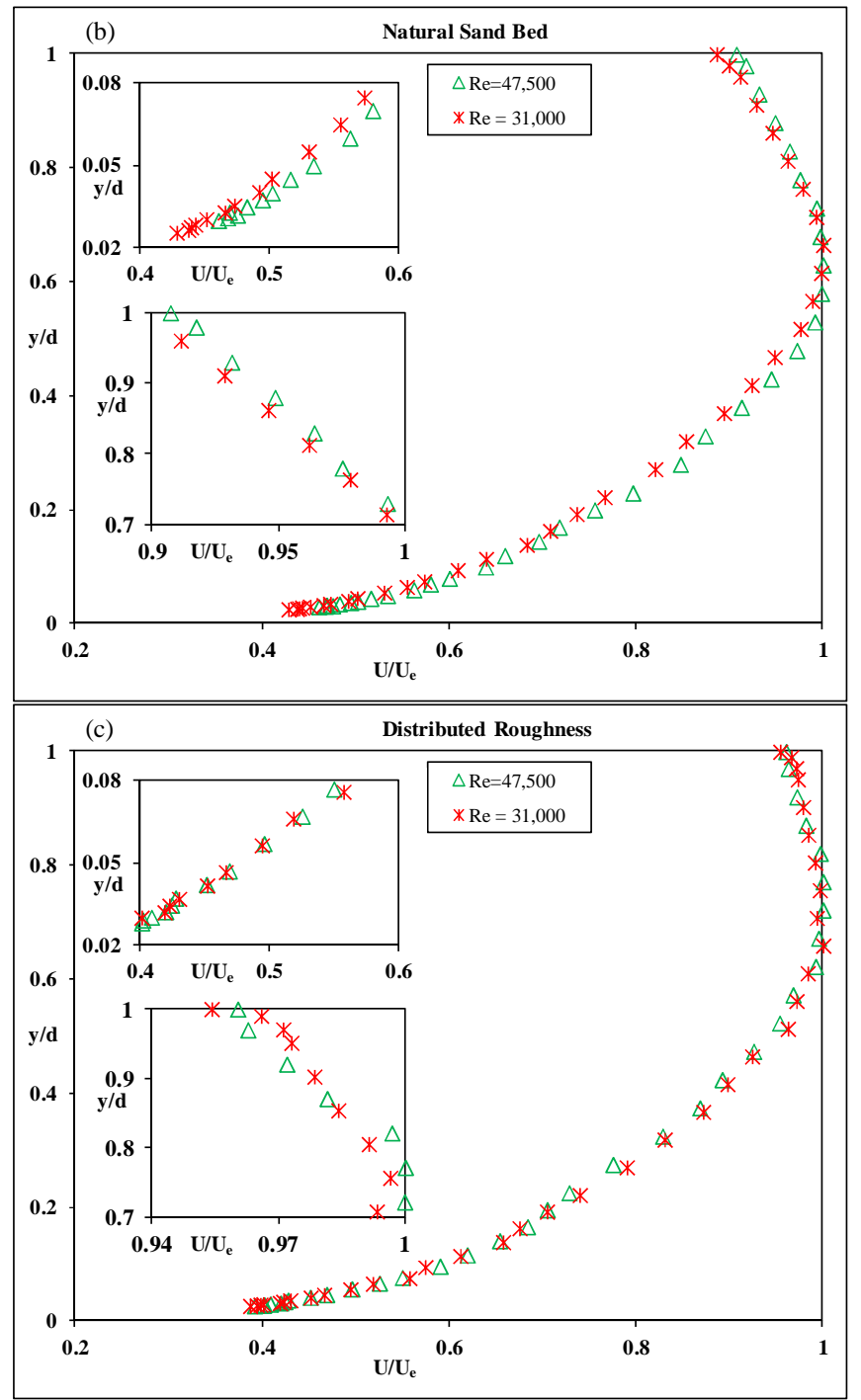

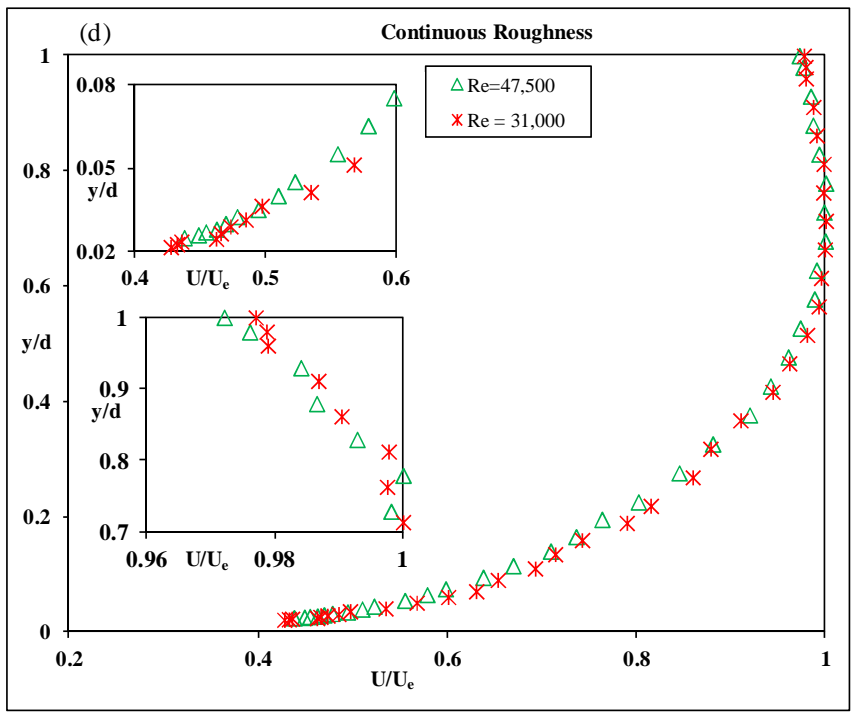

Figure 4: Streamwise Mean Velocity Profile - Outer Scaling 
One can also note from Figure 4 that the location of maximum velocity moves upward from the bed with the variation of roughness. Location of maximum velocity for the distributed roughness and continuous roughness is at $y / d$ $\sim 0.8$ from the bed for higher Reynolds number but the location of the same is at $y / d \sim 0.7$ for lower Reynolds number. However, the location of maximum velocity for the flow over smooth bed and sand bed does not vary much with the variation of Reynolds number.

\subsection{Mean Velocity Profile: Inner Scaling}

The distributions of the streamwise component of the mean velocity in inner scaling are shown in Figure 5. Friction velocity were calculated using the Clauser method by fitting the mean velocity profiles with the classical log law, $\mathrm{U}^{+}=\kappa^{-1} \ln \mathrm{y}^{+}+\mathrm{B}-\Delta \mathrm{U}^{+}$. Here, $\mathrm{U}^{+}=\mathrm{U} / \mathrm{U}_{\tau}, \mathrm{y}^{+}=\mathrm{y}_{\tau} / \mathrm{v}$, $\kappa=0.41$ and $\mathrm{B}=5$ are log-law constants and $\Delta \mathrm{U}^{+}$is the roughness function representing the downward shift of the velocity profile. $\Delta \mathrm{U}^{+}$is zero for flow over the smooth bed. The smooth bed test data agrees well with the standard loglaw (solid line). The profiles collapse at each Reynolds number in the viscous sub layer as well as overlap region. As Reynolds number increases, the extent over which the experimental data follow the log law increases. As the Reynolds number decreases the length of log layer also decreases. The expected downward-right shift of the profile is clearly visible for the rough beds (Figure $5 \mathrm{~b}$ to $5 \mathrm{~d}$ ). Since the downward shift of the profile is a measure of the effect of roughness, so it increases from natural sand bed to continuous roughness bed to distributed roughness bed.

Friction coefficient is calculated as, $\mathrm{C}_{\mathrm{f}}=2\left(\mathrm{U}_{\tau} / \mathrm{U}_{\mathrm{e}}\right)^{2}$ and found to be maximum for distributed roughness followed by continuous roughness and sand bed. It was also found that friction coefficient reduces as the Reynolds number increases. One can also note that the flow over the natural sand bed resulted in a reduction in friction coefficient compared to the flow over the impermeable rough surfaces (distributed roughness and continuous roughness). The probable explanation can be the development of finite slip velocity at the interface of the permeable layer which will eventually reduce the surface friction. However, Zagni and Smith (1976) noted that the boundary resistance of the permeable boundaries is higher than that of nonpermeable boundaries having identical rugosity and thought that it might be due to the net effect of combined loss of energy (energy dissipation within the transition zone in the porous medium and additional energy loss due to fluid and momentum exchange across the interface translated back into the main flow).

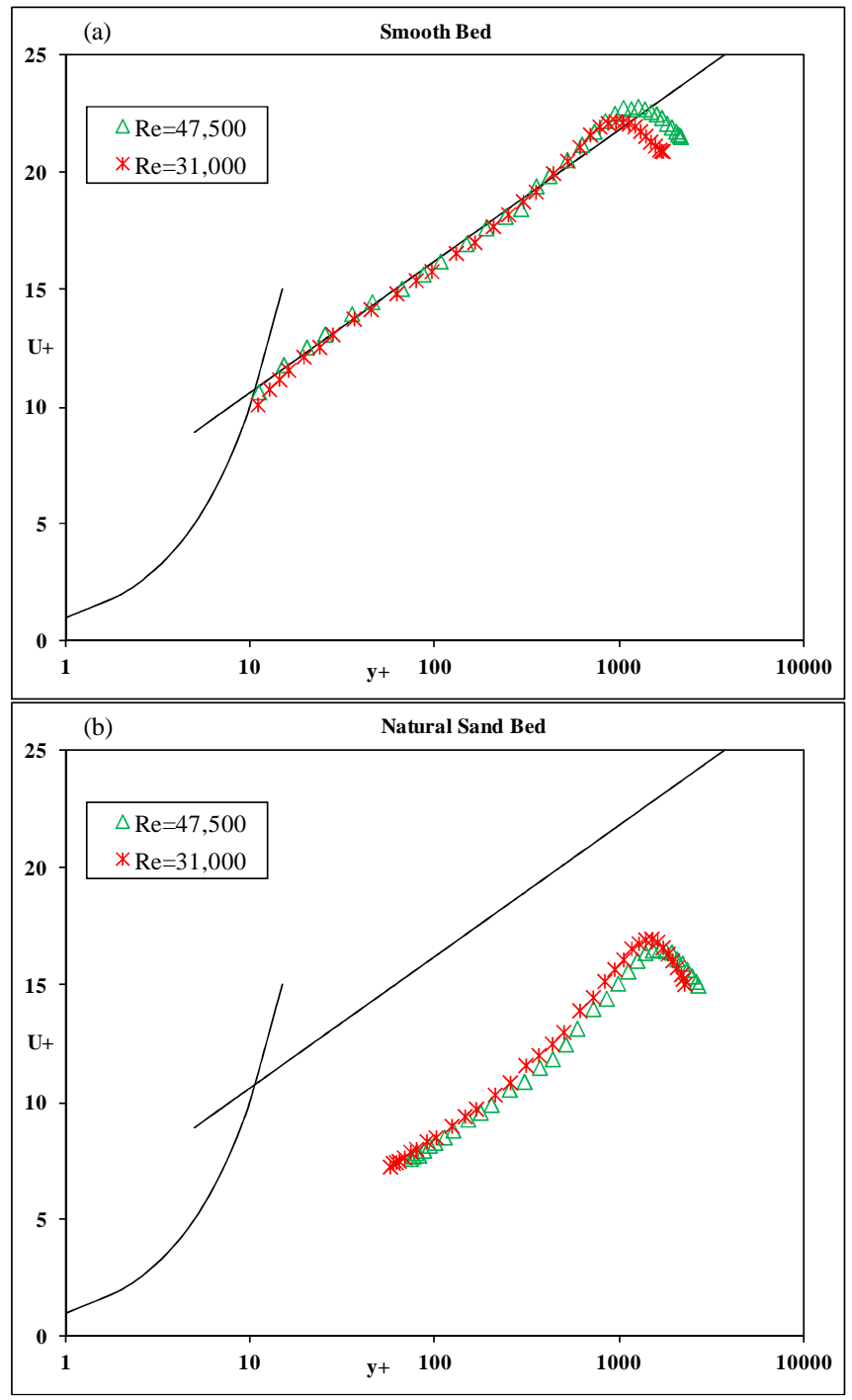




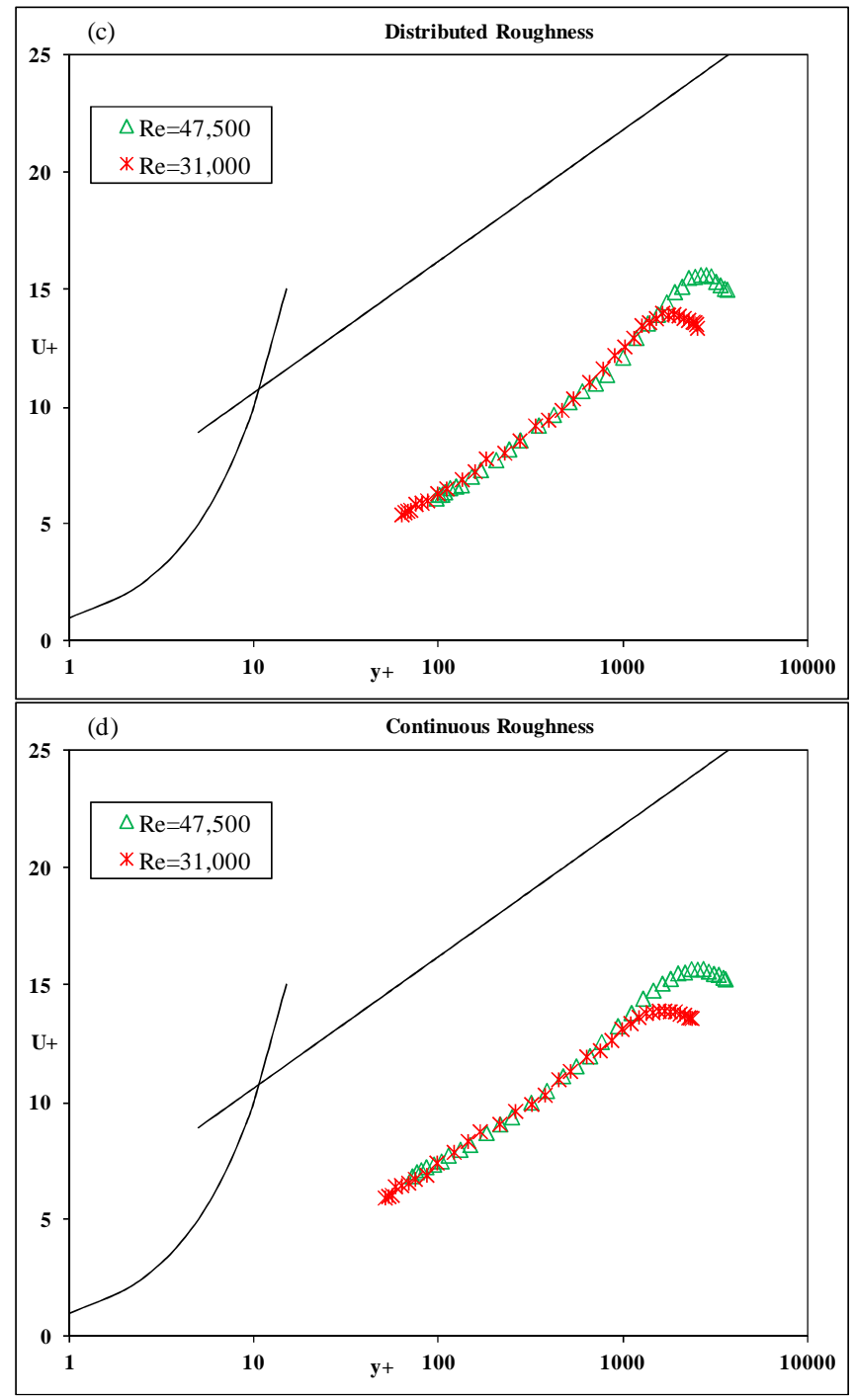

Figure 5: Streamwise Mean Velocity Profile - Inner Scaling

\section{CONCLUSIONS}

The present study was carried out to understand the extent of the effects of roughness and Reynolds number on mean velocity and velocity profile. To this end, four different types of bed surface conditions and two different Reynolds number values were adopted in the study. The main findings are summarized as follows:

1. Mean streamwise velocity for flow over smooth bed collapses reasonably for different Reynolds numbers.

2. Higher Reynolds number causes the increased velocity in the near bed location and simultaneously causes the reduction in velocity near the free surface for smooth bed and sand bed.

3. There is no effect of Reynolds number for flow near the bed or near the free surface for the flow over the distributed roughness and continuous roughness.

4. In the outer region, each velocity profile shows a slight dip where the local maximum value occurs below the free surface and $\delta \mathrm{U} / \delta \mathrm{y}$ is negative in the vicinity of the free surface.
5. Although the velocity dip is largest for the sand bed, the smooth surface shows a greater dip than both the continuous roughness and the distributed roughness.

6. The location of maximum velocity moves upward from the bed with the variation of roughness.

7. Location of maximum velocity is closer to free surface for the distributed roughness and continuous roughness for higher Reynolds number.

8. The location of maximum velocity for the flow over smooth bed and sand bed does not vary much with the variation of Reynolds number.

9. The smooth bed test data agrees well with the standard log-law. The profiles collapse at various Reynolds number in the viscous sub layer as well as overlap region.

10. As Reynolds number increases, the extent over which the experimental data collapse onto log law increases.

11. As the Reynolds number is decreasing the length of $\log$ layer is getting reduced.

12. As the downward shift of the profile is a measure of the effect of roughness, the distributed roughness shows a maximum effect, followed by continuous roughness and natural sand bed.

13. Friction coefficient is found maximum for distributed roughness followed by continuous roughness and sand bed.

14. Friction coefficient reduces as the Reynolds number increases.

15. Flow over the natural sand bed resulted in a reduction in friction coefficient compared to the flow over the impermeable rough surfaces.

\section{REFERENCES}

[1]. Afzal, B., Faruque, M. A. A., and Balachandar, R. (2009). "Effect of Reynolds number, near-wall perturbation and turbulence on smooth open channel flows." Journal of Hydraulic Research, 47(1), 66-81.

[2]. Balachandar, R., and Patel, V. C. (2002). "Rough wall boundary layer on plates in open channels." Journal of Hydraulic Engineering, 128(10), 947-951.

[3]. Bey, A., Faruque, M. A. A., and Balachandar, R. (2007). "Two dimensional scour hole problem: Role of fluid structures." Journal of Hydraulic Engineering, 133(4), 414430.

[4]. Faruque, M. A. A., Sarathi, P., and Balachandar, R. (2006). "Clear water local scour by submerged threedimensional wall jets: Effect of tailwater depth." Journal of Hydraulic Engineering, 132(6), 575-580.

[5]. Kirkgöz, M. S., and Ardiçhoğlu, M. (1997). "Velocity profiles of developing and developed open channel flow." Journal of Hydraulic Engineering, 123(2), 1099-1105.

[6]. Nezu, I. (2005). "Open-channel flow turbulence and its research prospect in the $21^{\text {st }}$ century." Journal of Hydraulic Engineering, 131(4), 229-246.

[7]. Sarathi, P., Faruque, M. A. A., and Balachandar, R. (2008). "Scour by submerged square wall jets at low densimetric Froude numbers." Journal of Hydraulic Research, 46(2), 158-175. 
[8]. Schlichting, H. (1979). Boundary-Layer theory. McGraw-Hill Classic Textbook Reissue Series, McGrawHill, Inc., United States of America.

[8]. Tachie, M. F., Bergstrom, D. J., and Balachandar, R. (2003). "Roughness effects in low- $R e_{\square}$ open-channel turbulent boundary layers." Experiments in Fluids, 35, 338346.

[9]. Zagni, A. F. E. and Smith, K. V. H. (1976). "Channel flow over permeable beds of graded spheres." Journal of Hydraulics Division, Proceedings of the ASCE, 102 (HY2), 207-222. 